1 Universidade do Estado do Rio de Janeiro (Uerj), Instituto de Medicina Social (IMS) - Rio de Janeiro (RJ) - Brasil. carinne.mag@gmail.com

2 Universidade Federal de Minas Gerais (UFMG), Faculdade de Medicina, Departamento de Medicina Preventiva - Belo Horizonte (MG), Brasil.

\section{PET-Saúde/GraduaSUS na visão de atores do serviço e do ensino: contribuições, limites e sugestões}

\author{
PET-Health/GraduaSUS in the perspective of service and teaching \\ actors: contributions, limits, and suggestions
}

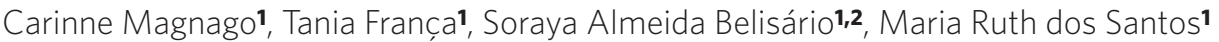

DOI: 10.1590/0103-11042019S102

RESUMO Este manuscrito apresenta os resultados de um survey on-line conduzido com os coordenadores de projetos e de grupos da última edição do Programa de Educação pelo Trabalho para a Saúde (PET-Saúde/GraduaSUS), que buscou identificar os resultados alcançados e apontar os limites e as contribuições dessa edição na produção de mudanças. O survey foi realizado em março e abril de 2018, com 445 coordenadores, cujos dados quantitativos foram tratados estatisticamente; e os qualitativos, por análise de conteúdo, dando origem a quatro categorias: As repercussões positivas do PET-Saúde/GraduaSUS; Os aspectos negativos do PET-Saúde/ GraduaSUS; A coordenação dos projetos pelo serviço de saúde: ponto controverso; e Sugestões de mudanças. Constatou-se que o PET-Saúde/GraduaSUS contribuiu para o desenvolvimento profissional, ampliação e diversificação dos cenários de aprendizagem, fortalecimento da atenção primária e da educação interprofissional nos currículos e beneficiou a comunidade local. Os principais aspectos positivos da edição foram a coordenação de projeto pelo serviço de saúde e os relatos de experiências na Comunidade de Práticas; já a composição numérica e uniprofissional dos grupos foi apontada como aspecto negativo.

PALAVRAS-CHAVE Serviços de integração docente-assistencial. Educação continuada. Educação superior. Políticas públicas de saúde. Desenvolvimento de pessoal.

ABSTRACT This manuscript presents the results of an online survey conducted with the project and group coordinators of the last edition of the Education through Work for Health Program (PETHealth/GraduaSUS), which sought to identify the results achieved and to point out the limits and contributions of such edition for the production of changes. The survey was carried out in March and April 2018, with 445 coordinators, whose quantitative data were treated statistically; and qualitative data by content analysis, giving rise to four categories: The positive repercussions of PET-Health/GraduaSUS; The negative aspects of PET-Health/GraduaSUS; The coordination of the projects by the health service: controversial point; and Suggestions for changes. It was found that the PET-Health/GraduaSUS contributed to the professional development, expansion and diversification of the learning scenarios, strengthening primary care and interprofessional education in the curricula and benefited the local community. The main positive aspects of the edition 
were the coordination of the project by the health service and the reports of experiences in the Community of Practices; the numerical and uniprofessional composition of the groups, however, was pointed as a negative aspect.

KEYWORDS Teaching care integration services. Education, continuing. Education, higher. Public health policy. Staff development.

\section{Introdução}

O Programa de Educação pelo Trabalho para a Saúde (PET-Saúde), constituído em 2008 pelo Ministério da Saúde (MS) em parceria com o Ministério da Educação, tem por principal objetivo induzir mudanças no processo de formação de profissionais de saúde, em alinhamento às necessidades do Sistema Único de Saúde (SUS)'.

Nessa perspectiva, assume como fio condutor o desenvolvimento de atividades baseadas na integração ensino-serviço-comunidade e orientadas pelas Diretrizes Curriculares Nacionais (DCN), que estimulem a vivência de estudantes nos serviços de saúde por meio de grupos de aprendizado tutorial, conformados por tutores, preceptores e acadêmicos ${ }^{\mathbf{1 , 2}}$.

Inicialmente direcionado para a Estratégia Saúde da Família (ESF), o PET-Saúde foi posteriormente estendido, por meio de edições temáticas, para outras áreas consideradas prioritárias para o SUS: vigilância em saúde; saúde mental e Redes de Atenção à Saúde ${ }^{3}$. Em 2015, lançada a edição GraduaSUS, o programa assumiu como foco o currículo das graduações em saúde, a qualificação dos processos de integração e a formação de preceptores e docentes ${ }^{4}$. Ao longo desses dez anos de programa, foram publicados 8 editais e desenvolvidos quase 800 projetos em todas as regiões do País ${ }^{3}$.

A edição GraduaSUS, objeto de estudo deste manuscrito, foi configurada no sentido de aproximar o PET-Saúde com as demais políticas nacionais de educação na saúde em vigência e de superar os desafios constatados em edições anteriores. Tendo isso em vista, a referida edição estabelece, entre outros pontos, que a coordenação dos projetos seja realizada pelas secretarias de saúde, função relegada às instituições de ensino nas edições anteriores; a obrigatoriedade de participação do curso de medicina; e a constituição dos grupos tutoriais por curso de graduação, em oposição à lógica multiprofissional prevista anteriormente. Em dois anos de vigência, envolveu 5.893 atores bolsistas e voluntários, entre estudantes, profissionais de saúde e docentes de 105 diferentes projetos construídos coletivamente $\mathbf{3}^{\mathbf{3} 4}$.

Considerando a finalização da vigência da última edição e a escassez de pesquisas analíticas sobre o programa, este manuscrito apresenta os resultados de um survey conduzido com os coordenadores de projetos e de grupos do PETSaúde/GraduaSUS. Desenvolvido com o intuito de atender ao item relativo ao monitoramento e avaliação dos projetos constante no edital que rege a edição $0^{4}$, o survey buscou identificar os resultados alcançados com o desenvolvimento das propostas e apontar os limites e as contribuições do PET-Saúde/GraduaSUS na produção de mudanças na formação em saúde e na qualificação dos serviços de saúde.

\section{Métodos}

Trata-se de estudo descritivo e exploratório, de abordagem mista, desenvolvido em março e abril de 2018, por meio de um survey on-line. Esse método de coleta de dados é 
frequentemente utilizado em pesquisas de grande escala, visando obter as opiniões de um determinado público-alvo mediante um questionário, neste caso, autoaplicável e disponibilizado virtualmente.

A população do estudo foi composta por todos os coordenadores do PET-Saúde/GraduaSUS, sendo 105 coordenadores de projeto, os quais são obrigatoriamente vinculados aos serviços de saúde, e 394 coordenadores de grupos, função exercida por profissionais das Instituições de Ensino Superior (IES). O convite para participação na pesquisa foi realizado a todos, por meio de correio eletrônico contendo o link de acesso ao survey e o prazo final para o seu preenchimento.

Constituíram a amostra todos os 445 (89,1\%) respondentes, dos quais 103 coordenadores de projeto $(98,1 \%)$ e 388 coordenadores de grupo (88,1\%), alcançando-se coberturas superiores a $80 \%$ em todos as regiões onde se localizam as secretarias de saúde e as IES que aderiram ao PET-Saúde/GraduaSUS.

Para a elaboração do survey, um instrumento utilizado anteriormente pelo Departamento de Gestão da Educação na Saúde da Secretaria de Gestão do Trabalho e da Educação na Saúde do MS (Deges/SGTES/MS) para o monitoramento do PET-Saúde foi adaptado ao contexto da edição GraduaSUS ${ }^{5}$, dando origem a um questionário estruturado com 34 questões agrupadas em quatro blocos, posteriormente configurado em formulário eletrônico no FormSUS. Além dessas questões, um campo de livre preenchimento foi deixado ao fim do instrumento para o registro voluntário dos respondentes quanto às suas impressões sobre a edição em análise.

As questões do bloco 1 buscaram identificar a escolarização, a formação e o tempo na instituição dos sujeitos pesquisados. $\mathrm{O}$ bloco 2 agrupou questões pertinentes à adequação da estrutura física dos serviços de saúde para o desenvolvimento das atividades do projeto, os tipos de atividades desenvolvidas e os mecanismos utilizados para a seleção dos participantes do PET-Saúde/GraduaSUS. O bloco 3 foi composto por questões que buscaram identificar os principais resultados alcançados pelos projetos vis-à-vis os objetivos do programa previstos em edital. Por fim, o bloco 4 objetivou conhecer as opiniões dos coordenadores sobre a pertinência do formato da edição do PET-Saúde/GraduaSUS em relação às demais edições e oportunizar o registro de pontos positivos, negativos, sugestões de mudanças e dificuldades enfrentadas na condução do programa.

Os dados quantitativos foram analisados por estatística descritiva via Microsoft Excel $^{\circledR}$ e estão apresentados sob o formato de tabelas. Os dados qualitativos, derivados do campo livre, foram submetidos à análise de conteúdo proposta por Bardin 6 . Para tanto, os registros foram organizados, submetidos à leitura exaustiva e, posteriormente, codificados e categorizados por similaridade semântica, resultando em quatro categorias analíticas: As repercussões positivas do PET-Saúde/GraduaSUS; Os aspectos negativos do PET-Saúde/GraduaSUS; A coordenação dos projetos pelo serviço de saúde: ponto controverso; e Sugestões de mudanças.

Em atendimento às normas previstas na Resolução no 466/2012 do Conselho Nacional de Saúde, o projeto foi submetido e aprovado pelo Comitê de Ética em Pesquisa do Instituto de Medicina Social da Universidade do Estado do Rio de Janeiro, sob o Parecer 2.503.706.

\section{Resultados e discussão}

Destaca-se a alta preponderância de graduados em enfermagem na coordenação de projetos $(n=40 ; 38,8 \%)$ e de grupos ( $n=88 ; 25,7 \%)$; e a baixa participação de médicos na coordenação dos grupos ( $n=43 ; 12,6 \%)$, não obstante à obrigatoriedade deste curso para aprovação dos projetos.

Ademais, verificou-se alta escolarização no conjunto dos respondentes $(n=445)$, dos quais $83,4 \%$ possuem o mestrado ou doutorado como o maior nível de formação. Em se tratando do tempo de serviço, a maioria dos coordenadores de projeto $(54,4 \%)$ e de curso $(43,3 \%)$ está inserida há mais de dez anos, respectivamente, nas secretarias de saúde e nas IES participantes do PET-Saúde. 
Verifica-se, na tabela 1 , que a estrutura física dos serviços de saúde foi considerada adequada para o desenvolvimento das atividades do programa por $91,8 \%$ dos coordenadores de grupo e por mais de $55 \%$ dos coordenadores de projeto, sendo as atividades de ensino as mais desenvolvidas; e as de assistência, as menos desenvolvidas. Esses achados vão de encontro ao que sugere grande parte dos estudos que se debruçam sobre o tema ensino na saúde, os quais apontam que a infraestrutura inadequada dos cenários de prática é um dos principais fatores que limitam a condução de atividades de ensino-aprendizagem na realidade do SUS ${ }^{7-9}$.

A realização de atividades por demanda do serviço/comunidade, mesmo que não previstas inicialmente no projeto, foi apontada por mais de 90\% dos coordenadores de projeto e de grupo; e mais de $80 \%$ deles afirmaram que as atividades foram realizadas conjuntamente pelas IES e serviços de saúde (tabela 1).

No que tange às modalidades de seleção de participantes bolsistas do PET-Saúde/ GraduaSUS, as opções mais frequentemente utilizadas, segundo o conjunto de respondentes, foram o processo seletivo mediado por edital para a escolha dos alunos; e o reconhecimento/ experiência profissional para a seleção dos coordenadores de grupo e de tutores/preceptores. Para a maioria deles, os projetos também contaram com alunos e tutores/preceptores em caráter de voluntariado (tabela 1).

É interessante destacar que, nos dois anos de funcionamento da edição GraduaSUS, cerca de 1.700 estudantes participaram como voluntários do programa, ou seja, sua participação não esteve condicionada ou motivada apenas ao recebimento da bolsa oferecida. A magnitude dessa adesão demonstra a importância do programa na comunidade estudantil, assim como entre os profissionais e docentes da saúde, os quais também atuaram no programa como preceptores $(\mathrm{n}=307)$ e tutores voluntários $(\mathrm{n}=191)^{3}$.

Tabela 1. Aspectos relacionados com o desenvolvimento das atividades dos projetos e mecanismos de seleção dos participantes do Programa de Educação para o Trabalho em Saúde

\begin{tabular}{|c|c|c|c|c|}
\hline \multirow[t]{2}{*}{ Variáveis analisadas } & \multicolumn{2}{|c|}{ Coordenadores de projeto } & \multicolumn{2}{|c|}{ Coordenadores de grupo } \\
\hline & $n=103$ & $\%$ & $n=342$ & $\%$ \\
\hline \multicolumn{5}{|c|}{ Adequação da estrutura física dos serviços de saúde para as atividades } \\
\hline Não & 6 & 5,8 & 2 & 0,6 \\
\hline Parcialmente & 39 & 37,9 & 26 & 7,6 \\
\hline Sim & 58 & 56,3 & 314 & 91,8 \\
\hline \multicolumn{5}{|l|}{ Tipos de atividades desenvolvidas } \\
\hline Assistência & 70 & 68,0 & 209 & 61,1 \\
\hline Ensino & 97 & 94,2 & 320 & 93,6 \\
\hline Extensão & 88 & 85,4 & 305 & 89,2 \\
\hline Pesquisa & 85 & 82,5 & 273 & 79,8 \\
\hline \multicolumn{5}{|c|}{ Desenvolvimento de atividades não previstas no projeto, por necessidade dos serviços de saúde/comunidade } \\
\hline Não sei responder & 0 & 0,0 & 6 & 1,8 \\
\hline $\begin{array}{l}\text { Não, as atividades restringiram-se ao projeto original e ao } \\
\text { previsto no currículo }\end{array}$ & 5 & 4,9 & 25 & 7,3 \\
\hline Sim, de forma eventual & 38 & 36,9 & 144 & 42,1 \\
\hline Sim, frequentemente & 60 & 58,3 & 167 & 48,8 \\
\hline
\end{tabular}


Tabela 1. (cont.)

\begin{tabular}{|c|c|c|c|c|}
\hline \multicolumn{5}{|c|}{ Desenvolvimento conjunto das atividades pelos serviço de saúde e instituições de ensino } \\
\hline $\operatorname{sim}$ & 88 & 85,4 & 280 & 81,9 \\
\hline Parcialmente & 14 & 13,6 & 62 & 18,1 \\
\hline Não & 1 & 1 & 0 & 0 \\
\hline \multicolumn{5}{|c|}{ Mecanismos utilizados para seleção de alunos bolsistas } \\
\hline Processo seletivo via Edital & 91 & 88,3 & 312 & 91,2 \\
\hline Coeficiente de rendimento escolar & 10 & 9,7 & 64 & 18,7 \\
\hline Por adesão & 7 & 6,8 & 33 & 9,6 \\
\hline Indicação & 11 & 10,7 & 16 & 4,7 \\
\hline Não sei responder & 5 & 4,9 & 0 & 0,0 \\
\hline \multicolumn{5}{|c|}{ Mecanismos utilizados para seleção de tutores/preceptores } \\
\hline Indicação & 52 & 50,5 & 119 & 34,8 \\
\hline Reconhecimento/experiência profissional & 61 & 59,2 & 227 & 66,4 \\
\hline Processo seletivo via Edital & 32 & 31,1 & 97 & 28,4 \\
\hline Por adesão & 26 & 25,2 & 111 & 32,5 \\
\hline Nenhuma das alternativas anteriores & 1 & 1,0 & 3 & 0,9 \\
\hline Não sei responder & 2 & 1,9 & 4 & 1,2 \\
\hline \multicolumn{5}{|c|}{ Mecanismos utilizados para seleção de coordenadores de grupo } \\
\hline Indicação & 41 & 39,8 & 109 & 31,9 \\
\hline Reconhecimento/experiência profissional & 70 & 68,0 & 252 & 73,7 \\
\hline Por adesão & 27 & 26,2 & 100 & 29,2 \\
\hline Processo seletivo via Edital & 6 & 5,8 & 13 & 3,8 \\
\hline Nenhuma das alternativas anteriores & 2 & 1,9 & 6 & 1,8 \\
\hline Não sei responder & 3 & 2,9 & 4 & 1,2 \\
\hline \multicolumn{5}{|l|}{ Existência de voluntários } \\
\hline Aluno & 101 & 98,1 & 329 & 96,2 \\
\hline Preceptor & 53 & 51,5 & 136 & 39,8 \\
\hline Tutor & 53 & 51,5 & 136 & 39,8 \\
\hline Não há voluntários & 2 & 1,9 & 7 & 2,0 \\
\hline
\end{tabular}

Fonte: Elaboração própria a partir dos dados da pesquisa, 2018.

Na tabela 2, verifica-se que, de acordo com a maior parte dos respondentes, os objetivos primários do PET-Saúde/GraduaSUS foram alcançados, especialmente o que indica 'promover a qualificação da integração ensino-serviço-comunidade', considerada pilar do movimento de mudanças da formação dos profissionais de saúde no Brasil.

A integração ensino-serviço-comunidade é compreendida como um trabalho coletivo, que integra os diferentes atores do quadrilátero da formação em saúde ${ }^{10}$, na direção do 
desenvolvimento de atividades articuladas e coerentes à realidade local, que conduzam, ao mesmo tempo, à qualificação da assistência prestada ao usuário, à excelência da formação e à educação permanente dos trabalhadores da saúde. Configura-se, portanto, como alicerce da construção de novos modos de aprender, ensinar e fazer saúde, contribuindo para ampliação da capacidade crítica e reflexiva dos atores e melhoria da qualidade de vida da população ${ }^{11,12}$.

Nessa perspectiva, com exceção de um, todos os respondentes afirmaram que a edição GraduaSUS contribuiu positivamente ou muito positivamente para o desenvolvimento profissional; e mais de $80 \%$ dos coordenadores de projeto e de grupo afirmaram que as atividades desenvolvidas no âmbito do PET-Saúde/ GraduaSUS contribuíram para o desenvolvimento da educação interprofissional e práticas colaborativas, promoveram a ampliação e/ou diversificação dos cenários de aprendizagem e beneficiaram a comunidade local (tabela 2).

Segundo os respondentes, também foram verificadas repercussões positivas nos serviços do SUS e nas unidades curriculares das instituições de ensino. Ademais, o envolvimento da comunidade no decorrer do projeto foi observado ou parcialmente observado, nesta ordem, por $81,6 \%$ e $74,9 \%$ dos coordenadores de projeto e de grupo (tabela 2).

Esses achados indicam que a edição do PET-Saúde em análise avançou no que tange às implicações nos serviços de saúde e na comunidade e robustecem a importância da parceria interinstitucional e da participação da população nos espaços de deliberação e de tomada de decisão que envolvem o SUS, nos moldes da gestão participativa ${ }^{13}$. Sugerem, ainda, que programas como o PET-Saúde são capazes de catalisar a inserção do debate e de atividades baseadas na educação interprofissional no SUS, subsidiando o rompimento do modelo hegemônico de formação em saúde ${ }^{\mathbf{1 4}}$.

Tabela 2. Resultados alcançados pelos projetos do Programa de Educação para o Trabalho em Saúde edição GraduaSUS, segundo os coordenadores. Brasil, 2018

\begin{tabular}{|c|c|c|c|c|}
\hline \multirow[t]{2}{*}{ Variáveis analisadas } & \multicolumn{2}{|c|}{ Coordenadores de projeto } & \multicolumn{2}{|c|}{ Coordenadores de grupo } \\
\hline & $n=103$ & $\%$ & $n=342$ & $\%$ \\
\hline \multicolumn{5}{|l|}{ Objetivos alcançados com o PET-Saúde/GraduaSUS } \\
\hline $\begin{array}{l}\text { Favoreceu mudanças curriculares alinhadas às Diretrizes } \\
\text { Curriculares Nacionais }\end{array}$ & 79 & 76,7 & 266 & 77,8 \\
\hline $\begin{array}{l}\text { Promoveu a qualificação do processo de integração ensino- } \\
\text {-serviço-comunidade }\end{array}$ & 101 & 98,1 & 324 & 94,7 \\
\hline $\begin{array}{l}\text { Promoveu articulações com outros projetos de integração } \\
\text { ensino-serviço-comunidade nos territórios }\end{array}$ & 95 & 92,2 & 298 & 87,1 \\
\hline \multicolumn{5}{|l|}{ Contribui para o desenvolvimento e formação profissional } \\
\hline Muito positivamente & 78 & 75,7 & 271 & 79,2 \\
\hline Positivamente & 24 & 23,3 & 71 & 20,8 \\
\hline Não interfere & 1 & 1,0 & 0 & 0,0 \\
\hline Prejudica & 0 & 0,0 & 0 & 0,0 \\
\hline \multicolumn{5}{|c|}{ Promoveu a ampliação/diversificação de cenários de ensino-aprendizagem } \\
\hline Sim & 88 & 85,4 & 298 & 87,1 \\
\hline Parcialmente & 14 & 13,6 & 40 & 11,7 \\
\hline Não & 1 & 1,0 & 4 & 1,2 \\
\hline
\end{tabular}


Tabela 2. (cont.)

\begin{tabular}{|c|c|c|c|c|}
\hline \multicolumn{5}{|c|}{ Contribuiu para os serviços do Sistema Único de Saúde } \\
\hline Sim & 82 & 79,6 & 279 & 81,6 \\
\hline Parcialmente & 20 & 19,4 & 58 & 17,0 \\
\hline Não & 0 & 0,0 & 4 & 1,2 \\
\hline Não sei responder & 1 & 1,0 & 1 & 0,3 \\
\hline \multicolumn{5}{|c|}{ Fortaleceu a atenção primária nas unidades curriculares das instituições de ensino } \\
\hline $\operatorname{Sim}$ & 69 & 67,0 & 247 & 72,2 \\
\hline Parcialmente & 28 & 27,2 & 88 & 25,7 \\
\hline Não & 1 & 1,0 & 3 & 0,9 \\
\hline Não sei responder & 5 & 4,9 & 4 & 1,2 \\
\hline \multicolumn{5}{|c|}{ Beneficiou a comunidade local } \\
\hline $\operatorname{Sim}$ & 92 & 89,3 & 307 & 89,8 \\
\hline Parcialmente & 10 & 9,7 & 31 & 9,1 \\
\hline Não & 1 & 1,0 & 2 & 0,6 \\
\hline Não sei responder & 0 & 0,0 & 2 & 0,6 \\
\hline
\end{tabular}

Contribuiu para o processo de discussão ou de implantação do Contrato Organizativo de Ação Pública de EnsinoSaúde (Coapes)

\begin{tabular}{|c|c|c|c|c|}
\hline Sim, estamos em processo de discussão & 62 & 60,2 & 173 & 50,6 \\
\hline Sim, implantamos o Coapes & 15 & 14,6 & 50 & 14,6 \\
\hline $\begin{array}{l}\text { Não. Já utilizamos outro instrumento jurídico de contratua- } \\
\text { lização }\end{array}$ & 12 & 11,7 & 19 & 5,6 \\
\hline $\begin{array}{l}\text { Não. Adotamos apenas o termo de compromisso firmado } \\
\text { para o desenvolvimento do PET-Saúde/GraduaSUS }\end{array}$ & 7 & 6,8 & 48 & 14,0 \\
\hline Não sei responder & 7 & 6,8 & 52 & 15,2 \\
\hline
\end{tabular}

Contribuiu para o desenvolvimento da educação interprofissional e das práticas colaborativas

\begin{tabular}{|c|c|c|c|c|}
\hline $\operatorname{Sim}$ & 83 & 80,6 & 282 & 82,5 \\
\hline Parcialmente & 19 & 18,4 & 53 & 15,5 \\
\hline Não & 1 & 1,0 & 3 & 0,9 \\
\hline Não sei responder & 0 & 0,0 & 4 & 1,2 \\
\hline \multicolumn{5}{|c|}{ Envolvimento do controle/participação social no desenvolvimento do projeto } \\
\hline $\operatorname{Sim}$ & 40 & 38,8 & 109 & 31,9 \\
\hline Parcialmente & 44 & 42,7 & 147 & 43,0 \\
\hline Não & 16 & 15,5 & 71 & 20,8 \\
\hline Não sei responder & 3 & 2,9 & 15 & 4,4 \\
\hline
\end{tabular}

Fonte: Elaboração própria a partir dos dados da pesquisa, 2018.

Questionados especificamente se a edição GraduaSUS é inovadora ante as edições antecessoras, $77,3 \%$ do conjunto de coordenadores respondeu positivamente. Verificou-se, ainda, alto percentual dos que não souberam responder a esse questionamento, aludindo-se que tais respondentes podem não ter participado de edições anteriores (tabela 3 ). 
Tabela 3. Percepção dos coordenadores sobre a inovação do Programa de Educação para o Trabalho em Saúde edição GraduaSUS em relação às edições anteriores, segundo os coordenadores. Brasil, 2018

\begin{tabular}{lrrrr}
\hline Avaliação & \multicolumn{3}{c}{ Coordenadores de projeto } & Coordenadores de grupo \\
\cline { 2 - 5 } & $\mathbf{n = 1 0 3}$ & \% & $\mathbf{n = 3 4 2}$ & \% \\
\hline Sim & 81 & 78,6 & 263 & 76,9 \\
Não & 2 & 1,9 & 20 & 5,8 \\
Não sei responder & 20 & 19,4 & 59 & 17,3 \\
\hline
\end{tabular}

Fonte: Elaboração própria a partir dos dados da pesquisa, 2018

Na avaliação dos coordenadores, são dois os principais aspectos do PET-Saúde/GraduaSUS que impactaram positivamente na produção de mudanças no serviço e no ensino: a coordenação de projeto pelo serviço de saúde e os relatos de experiências na Comunidade de Práticas (CdP), dois grandes diferenciais dessa edição em relação às anteriores. $\mathrm{O}$ primeiro coloca o serviço de saúde como corresponsável pelo processo de mudanças operadas no campo da formação e como ator ativo na determinação e desenvolvimento das atividades operadas no âmbito do PET-Saúde 3 . O segundo aspecto se apresenta como uma oportunidade de compartilhamento de experiências e construção coletiva de conhecimento sobre o SUS. Por se tratar de uma rede virtual, a CdP rompe as barreiras geográficas e as dificuldades de encontros entre os atores que operam o SUS nos diferentes territórios, propiciando dinamismo na aprendizagem colaborativa.

Em relação aos principais aspectos negativos, a composição numérica dos grupos foi a mais indicada pelos coordenadores de projeto $(27,2 \%)$ e de grupo $(24,3 \%)$. Chama atenção que, para a maioria dos respondentes, nenhuma das opções apresentadas no questionário provocou impacto negativo na produção de mudanças, como exibido na tabela 4.

Tabela 4. Aspectos do Programa de Educação para o Trabalho em Saúde edição GraduaSUS que impactaram na produção de mudanças no ensino e no serviço de saúde, segundo os coordenadores. Brasil, 2018

\begin{tabular}{|c|c|c|c|c|}
\hline \multirow[t]{2}{*}{ Aspectos da edição do programa } & \multicolumn{2}{|c|}{ Coordenadores de projeto } & \multicolumn{2}{|c|}{ Coordenadores de grupo } \\
\hline & $n=103$ & $\%$ & $n=342$ & $\%$ \\
\hline \multicolumn{5}{|l|}{ Impactaram positivamente } \\
\hline Coordenação de projeto pelo serviço de saúde & 74 & 71,8 & 180 & 52,6 \\
\hline Relatos de experiências na Comunidade de Práticas & 60 & 58,3 & 181 & 52,9 \\
\hline Observância das diretrizes do Coapes & 46 & 44,7 & 148 & 43,3 \\
\hline Exigência obrigatória do curso de medicina & 39 & 37,9 & 92 & 26,9 \\
\hline Composição numérica dos grupos & 37 & 35,9 & 90 & 26,3 \\
\hline Nenhuma das opções anteriores provocaram impacto & 3 & 2,9 & 24 & 7,0 \\
\hline Não sei responder & 3 & 2,9 & 17 & 5,0 \\
\hline \multicolumn{5}{|l|}{ Impactaram negativamente } \\
\hline Coordenação de projeto pelo serviço de saúde & 10 & 9,7 & 74 & 21,6 \\
\hline Relatos de experiências na Comunidade de Práticas & 5 & 4,9 & 13 & 3,8 \\
\hline
\end{tabular}


Tabela 4. (cont.)

\begin{tabular}{lrrrr}
\hline Observância das diretrizes do Coapes & 3 & 2,9 & 10 & 2,9 \\
Exigência obrigatória do curso de medicina & 15 & 14,6 & 53 & 15,5 \\
Composição numérica dos grupos & 28 & 27,2 & 83 & 24,3 \\
Nenhuma das opções anteriores provocaram impacto & 53 & 51,5 & 146 & 42,7 \\
Não sei responder & 8 & 7,8 & 30 & 8,8 \\
\hline
\end{tabular}

Fonte: Elaboração própria a partir dos dados da pesquisa, 2018.

Para os coordenadores, em geral, os principais fatores que dificultaram a integração ensino-serviço-comunidade no âmbito do PET-Saúde/GraduaSUS foram a resistência dos profissionais dos serviços de saúde não participantes do programa e a comunicação. Parcela significante dos coordenadores de grupo $(33,3 \%)$ também indicou como aspecto dificultador a resistência dos gestores em liberar os profissionais para as atividades de ensino (preceptoria) (tabela 5).

Sobre a resistência dos profissionais de saúde para o exercício da preceptoria, estudos indicam que ela pode estar associada à incompatibilidade curricular com a lógica do serviço e à desvalorização desse papel por gestores, instituições de ensino e pelos próprios profissionais. Essa desvalorização perpassa pela ausência de regulamentação da função do preceptor, desconhecimento sobre a preceptoria e não bonificação pelas atividades de ensino desempenhadas ${ }^{15-17}$.

A preceptoria no contexto do PET-Saúde pode ser compreendida como atividade pedagógica exercida por profissional de saúde, que incorpora o ofício de mediar o processo de aprendizagem do estudante em contato com o mundo do trabalho. Nesse entendimento, instiga a problematização da realidade, a reflexão crítica e a busca por soluções coletivas que enfrentem as situações cotidianas do ensino-serviço ${ }^{\mathbf{1 4}}$. Pressupõe-se, portanto, que para o efetivo desempenho desse papel, fazem-se prementes processos de qualificação desses atores.

Pelo exposto, a edição GraduaSUS incluiu no seu rol de prerrogativas a exigência de os projetos contemplarem ações de desenvolvimento docente e preceptor, reafirmando que eles são indispensáveis para as mudanças que se pretende alcançar ${ }^{4}$.

Tabela 5. Fatores que dificultaram a integração ensino-serviço-comunidade no âmbito do Programa de Educação para o Trabalho em Saúde edição GraduaSUS, segundo os coordenadores. Brasil, 2018

\begin{tabular}{|c|c|c|c|c|}
\hline \multirow[t]{2}{*}{ Dificuldades na integração ensino-serviço } & \multicolumn{2}{|c|}{ Coordenadores de projeto } & \multicolumn{2}{|c|}{ Coordenadores de grupo } \\
\hline & $n=103$ & $\%$ & $n=342$ & $\%$ \\
\hline $\begin{array}{l}\text { Resistência dos profissionais dos serviços de saúde não } \\
\text { participantes do PET-Saúde/GraduaSUS }\end{array}$ & 41 & 39,8 & 149 & 43,6 \\
\hline $\begin{array}{l}\text { Resistência dos gestores em liberar os profissionais para as } \\
\text { atividades de ensino }\end{array}$ & 27 & 26,2 & 114 & 33,3 \\
\hline Comunicação & 38 & 36,9 & 104 & 30,4 \\
\hline $\begin{array}{l}\text { Pouca capacitação pedagógica do(s) preceptor(es) para } \\
\text { receber estudantes }\end{array}$ & 29 & 28,2 & 90 & 26,3 \\
\hline Relacionamento interpessoal & 25 & 24,3 & 73 & 21,3 \\
\hline Perfil inadequado do(s) estudante(s) & 7 & 6,8 & 9 & 2,6 \\
\hline Perfil inadequado do(s) tutor(es) acadêmicos & 8 & 7,8 & 16 & 4,7 \\
\hline
\end{tabular}




\begin{tabular}{lrrrr} 
Tabela 5. (cont.) & & & \\
\hline Resistência dos usuários & 1 & 1,0 & 10 & 2,9 \\
Rigidez nas propostas de trabalho & 4 & 3,9 & 17 & 5,0 \\
Nenhuma dificuldade foi encontrada & 19 & 18,4 & 76 & 22,2 \\
Não sei responder & 3 & 2,9 & 6 & 1,8 \\
\hline
\end{tabular}

Fonte: Elaboração própria a partir dos dados da pesquisa, 2018.

Quanto ao modelo de pagamento, os coordenadores foram unânimes em afirmar que o repasse direto de bolsas aos integrantes valoriza os sujeitos envolvidos no processo de integração ensino-serviço-comunidade; e, para a maioria $(76,4 \%)$, essa forma de repasse viabiliza, agiliza e fortalece o financiamento de ações de educação na saúde de acordo com as necessidades do SUS.

Em se tratando do campo de livre registro, 257 (57,8\%) coordenadores teceram comentários sobre a edição PET-Saúde/GraduaSUS, os quais foram agrupados nas quatro categorias apresentadas na sequência

\section{As repercussões positivas do PET-Saúde/GraduaSUS}

Os registros dos respondentes se referem sobremaneira às repercussões positivas do PET-Saúde/GraduaSUS, o qual foi considerado como um importante dispositivo de promoção da integração ensino-serviço-comunidade e de indução de mudanças curriculares alinhadas às DCN, em concordância com outros estudos sobre edições anteriores do programa ${ }^{18-20}$.

Oportunizou aos cursos vinculados momentos de discussão sobre formação, uma ampliação da integração ensino-serviço-comunidade e a certeza na construção de uma formação mais articulada no e para o SUS.

Contribuição de forma significativa para efetivar as mudanças curriculares que já vinham sendo construídas e ensaiadas em versões anteriores.
A nossa universidade conseguiu implantar, em 2018, uma disciplina interprofissional para todos os cursos da área da saúde nos moldes do PET-Saúde.

Ainda nessa perspectiva, o programa se configurou, de maneira significativa, como estratégia de ampliação dos cenários de prática, em especial para os cursos ainda pouco inseridos na APS, pelo número reduzido de Núcleos de Apoio à Saúde da Família e pouco reconhecimento da importância dessas profissões nesse nível de atenção ${ }^{3}$, como a fonoaudiologia, a biomedicina e medicina veterinária, proporcionando, em alguns casos, o primeiro contato de alunos desses cursos com a ESF.

Extrema importância para o grupo de Medicina Veterinária, pois colocou o aluno em contato com uma área extremamente importante e ao mesmo tempo pouco valorizada dentro do curso.

Na fisioterapia, os acadêmicos não possuem esse contato direto com o SUS. E, com o PET-Saúde, foi perceptivel a desenvoltura que os bolsistas adquiriram e o entendimento de como se trabaIha na rede SUS.

Cumpre destacar, ainda, que o PET-Saúde/ GraduaSUS foi pontuado como uma estratégia de mobilização da gestão universitária, culminando em alteração de normas internas e implementação de serviços de saúde vinculados às IES. Essas contribuições se alinham ao que apregoa a Resolução n $569 / 2017$, que ratifica a responsabilidade social das IES com o território no qual está inserido e sua comunidade e o 
compromisso em apoiar o desenvolvimento de serviços e redes assistenciais e com a formação de profissionais de saúde, transformando a realidade local ${ }^{21}$.

O PET-Saúde/GraduaSUS transformou a realidade da nossa universidade e dentre os legados posso ressaltar: 1) Promoveu curso de capacitação para farmacêuticos do SUS; 2) Promoveu a criação de um serviço de farmácia clínica.

Mobilização da gestão universitária e a realização de mudanças das normas internas que fortaleceram a preceptoria e as ações multiprofissionais.

\section{Os aspectos negativos do PET-Saúde/GraduaSUS}

Os coordenadores também chamaram atenção para os aspectos negativos da edição GraduaSUS, entre os quais, o mais citado foi a regra de composição uniprofissional dos grupos que limitou a integração entre os diferentes cursos da saúde.

Retrocesso os alunos e preceptores serem do mesmo curso, já que a integração curricular é almejada. O modelo não colaborou para as mudanças a que se propôs.

A não obrigatoriedade de os grupos serem interprofissionais prejudicou muito a proposta de integração dos cursos.

Segundo o edital que rege a edição GraduaSUS, a opção por grupos conformados por atores de um mesmo curso se assenta na ideia de expandir a discussão interna sobre o currículo, induzindo, portanto, mudanças efetivas nas grades curriculares. Não obstante, o mesmo edital preceitua a obrigatoriedade de atividades interprofissionais nos cenários de prática do SUS ${ }^{\mathbf{3 , 4}}$.

A obrigatoriedade dos cursos de medicina também foi ponto considerado negativo, pois, segundo os respondentes, a categoria apresenta resistência quanto às mudanças curriculares e à participação efetiva no desenvolvimento das atividades do projeto.

Entendo o objetivo que originou a obrigatória participação da medicina, no entanto, essa obrigatoriedade não garantiu a participação efetiva.

Muita resistência, principalmente do corpo docente [de medicina], tanto para reformar o currículo como para ampliar o cenário de prática.

Sobre isso, estudo realizado com escolas de medicina encontrou que a resistência dos docentes de medicina se configura como umas das principais dificuldades para implementação de mudanças curriculares ${ }^{22}$. Tal resistência pode estar relacionada com a crença dos docentes de que as mudanças, como a integração de disciplinas e a interprofissionalidade, poderiam aumentar sua carga de trabalho ${ }^{23}$.

A premissa que o ensino médico é mais resistente às modificações do que outras áreas encontra sustentação na própria historicidade das profissões da saúde. A medicina é a instituidora da prática de saúde científica e moderna e dela derivam os demais campos do saber da saúde, conjuntura que faz emergir a hegemonia médica perante outros profissionais de saúde. $\mathrm{O}$ médico ainda se percebe superior, e as demais categorias lutam para serem socialmente reconhecidas ${ }^{24-26}$.

Tal contexto vai de encontro às premissas da educação interprofissional e práticas colaborativas, que primam pelo rompimento do tribalismo das profissões mediante encontros de aprendizagem de diferentes categorias profissionais, para que possam aprender com, a partir de e sobre as outras para melhorar a colaboração profissional27.

A CdP, rede social colaborativa destinada aos atores do SUS, que foi adotada como plataforma de registro das experiências PETSaúde/GraduaSUS em caráter obrigatório, foi alvo de críticas em razão da sua instabilidade e da sua interface pouco responsiva, embora tenha sido considerada instrumento positivo para o compartilhamento de saberes. 
A Comunidade de Práticas: difícil de trabalhar, retorno demorado para a publicação.

A utilização da Plataforma Sabiá [sistema por meio do qual se acessa a Comunidade de Práticas] e da Comunidade de Práticas Muitos erros, muitos problemas. Inviabilidade na utilização, inclusive na busca por autores dos relatos.

Segundo os respondentes, outros aspectos relacionados com o PET-Saúde que limitaram as atividades e, por conseguinte, os resultados positivos dos projetos executados foram: o número reduzido de bolsas e o tempo de vigência da edição que foi considerado insuficiente para alcance das metas pactuadas.

Entre os fatores não diretamente associados ao formato da edição, citam-se: o despreparo e a rotatividade dos preceptores; as dificuldades de caráter logístico para encontros entre grupos de projetos interinstitucionais, conformados por meio de parceria entre IES de diferentes municípios; as dificuldades de cunho social, em especial, o aumento da violência das grandes metrópoles, que põem em risco à segurança de docentes e alunos; e os relacionados com a gestão municipal, conforme se evidencia nos trechos abaixo:

Mudança da gestão municipal, resultando em mudanças nas preceptorias dos grupos tutoriais que impactou, por consequência, na condução dos grupos.

Mudança de gestão, que dificultou a contratação de profissionais para a preceptoria, e a resistência do gestor em dar o atestado para autorização do pagamento de bolsas e em liberar os preceptores para as atividades do PET.

\section{A coordenação dos projetos pelo ser- viço de saúde: ponto controverso}

No âmbito do PET-Saúde/GraduaSUS, a função de coordenador de projeto deveria ser obrigatoriamente assumida por profissional vinculado à secretaria de saúde, sendo responsável pela organização e distribuição das atividades gerais do projeto e pelo fomento à integração dos grupos e ao desenvolvimento das atividades propostas.

Por seu caráter inédito, esse aspecto foi considerado por alguns como elemento facilitador e, por outros, como limitador da condução dos projetos.

O PET-Saúde GraduaSUS foi uma edição que, pela primeira vez, contou com o protagonismo do serviço, possibilitando, de fato, os graduandos vivenciarem e responderem às necessidades reais existentes do serviço.

Transferir a coordenação do projeto para o gestor emperrou a execução do projeto, que deveria estar sob a coordenação de um gestor universitário.

\section{Sugestões de mudanças}

Entre os registros dos coordenadores, foram encontradas sugestões de mudanças de pontos específicos da edição GraduaSUS e outras que visam, sobretudo, a continuidade e o fortalecimento do PET-Saúde, como: instituir o PET-Saúde como política permanente; lançar mais edições do programa; estender o tempo de vigência das edições; ampliar o número de cursos envolvidos; ampliar o número de alunos por grupos; prever a coordenação de projeto compartilhada entre a gestão e a IES; e o desenvolvimento de ações de monitoramento e avaliação in loco. Outras sugestões podem ser verificadas nos extratos abaixo:

O PET-Saúde enquanto política de integração ensino-serviço-comunidade deveria ter renovação automática, mediante critérios de avaliação, e recursos financeiros para apoiar a implementação das ações planejadas, a exemplo do Programa de Educação Tutorial do Ministério da Educação.

O edital poderia ser mais enxuto e focar em uma proposta de intervenção objetiva e específica, 
centrada na Educação Interprofissional, e sem a exigência do curso de medicina, pois nem todas as instituições que possuem cursos de saúde têm o curso de medicina.

O PET-Saúde, que assume a integração ensino-serviço-comunidade como dispositivo estruturante para a formação em saúde, tem somado contribuições ao movimento de aproximação do ensino ao sistema de saúde e de modificação da formação em saúde ${ }^{28}$.

\section{Considerações finais}

Este estudo procurou identificar os principais elementos de contribuição e de fragilidade do PETSaúde/GraduaSUS, objetivando instrumentalizar o gestor de saúde federal e subsidiar sua tomada de decisão no que tange aos desdobramentos do programa. Para tanto, considerou a percepção e opinião dos coordenadores de projeto (serviço) e de grupos (instituições de ensino), possibilitando apreender informações estratégicas sobre a condução dos projetos.

Os resultados do survey demonstraram a positividade, a magnitude e a relevância do programa na formação profissional dos atores envolvidos, bem como no cumprimento de seus principais objetivos - qualificar a integração ensino-serviço e promover mudanças curriculares dos cursos de saúde.

Considerada inovadora diante das às edições anteriores, o PET-Saúde/GraduaSUS aproximou as instituições de ensino aos serviços de saúde, os quais foram alçados a protagonistas e atores ativos na construção e desenvolvimento dos projetos. Esse ponto, em específico, foi majoritariamente considerado como um aspecto positivo da edição, mas alguns respondentes o compreenderam como elemento limitador. Essa aparente contradição pode se dever ao ineditismo dessa proposta ainda não totalmente absorvida pelos atores envolvidos, dado que, nas edições anteriores, a coordenação do projeto foi responsabilidade assumida pelas instituições formadoras.
As contribuições e sugestões sobre o PETSaúde/GraduaSUS sinalizam para a elaboração de futuros editais com investimento em ações de monitoramento e avaliação, retomada de composição dos grupos de natureza interprofissional, coordenação de projetos compartilhada entre atores do serviço e do ensino e de manutenção dos aspectos que norteiam as mudanças na lógica da formação dos profissionais e na dinâmica do trabalho em saúde.

Objetivando instrumentalizar os tomadores de decisão e subsidiar sua tomada de decisão no que tange aos desdobramentos do programa, os achados deste estudo foram apresentados e discutidos com o gestor de saúde federal, subsidiando a elaboração da nova edição do programa: PET-Saúde/Interprofissionalidade.

As transformações esperadas, justamente por envolverem mudanças de paradigmas e novas formas de agir e pensar saúde, não se concretizam rapidamente. O PET-Saúde/ GraduaSUS contribuiu enormemente para esse processo; e novos editais podem avançar na construção da integralidade da atenção, no fortalecimento da educação e do trabalho interprofissional e colaborativo e, por conseguinte, na melhoria dos resultados de saúde.

Na perspectiva de continuidade e instituição do programa como política permanente, novos estudos de monitoramento e avaliação precisam ser desenvolvidos para que seus dados subsidiem o aperfeiçoamento e fortalecimento dessa iniciativa que, como os resultados apresentados aqui sugerem, configura-se como um instrumento potente de produção de mudanças.

\section{Colaboradores}

Magnago C (0000-0001-8799-3225)* participou da concepção, planejamento, análise e interpretação dos dados, da elaboração do rascunho, revisão crítica do conteúdo e aprovação final do manuscrito. França T (0000-00028209-9811)* participou da concepção, planejamento, análise e interpretação dos dados; 
da elaboração do rascunho, revisão crítica do conteúdo e aprovação final do manuscrito. Belisário SA (0000-0002-2240-6146)* participou da concepção, planejamento, análise e interpretação dos dados; da elaboração do rascunho, revisão crítica do conteúdo e aprovação final do manuscrito. Santos MR (0000-00021669-206X)* participou da análise e interpretação dos dados; da elaboração do rascunho; da revisão crítica do conteúdo e aprovação final do manuscrito.

\section{Referências}

1. Brasil. Ministério da Saúde; Ministério da Educação. Portaria Interministerial $\mathrm{n}^{\mathrm{o}} 1.802$, de 26 de agosto de 2008. Institui o Programa de Educação pelo Trabalho para a Saúde - PET-Saúde. Diário Oficial da União. 27 Ago 2008.

2. Farias-Santos BCS, Noro LRA. PET-Saúde como indutor da formação profissional para o Sistema Único de Saúde. Ciênc. Saúde Coletiva [internet]. 2017 [acesso em 2018 jul 1]; 22(3):997-1004. Disponível em: http:// dx.doi.org/10.1590/1413-81232017223.15822016.

3. França T, Magnago C, Santos MR, et al. PET-Saúde/ GraduaSUS: retrospectiva, diferenciais e panorama de distribuição dos projetos. Saúde debate. 2018; 42(esp2):286-301.

4. Brasil. Ministério da Saúde, Secretaria de Gestão do Trabalho e da Educação na Saúde. Edital no 13, de 28 de setembro de 2015. Seleção para o Programa de Educação Pelo Trabalho Para a Saúde: PET-Saúde/ GraduaSUS - 2016/2017. Diário Oficial da União. 29 Set 2015.

5. Passarella MR. Programa de Educação pelo Trabalho para a Saúde - PET-Saúde/Saúde da Família: um estudo sobre a percepção dos participantes acerca da integração ensino-serviço-comunidade. [dissertação]. Rio de Janeiro: Escola Nacional de Saúde Pública Sergio Arouca; 2013. 172 p.

6. Bardin L. Análise de conteúdo. São Paulo: Edições $70 ; 2011$

7. Magnago C, Pierantoni CR, Carvalho CL, et al. Nurse training in health in different regions in Brazil. Rev. Bras. Saúde Mater. Infant. [internet]. 2017 [acesso em 18 jun. 2018]; 17(supl1):219-228. Disponível em: http://dx.doi.org/10.1590/1806-9304201700s100010.

8. Vieira SP, Pierantoni CR, Magnago C, et al. A graduação em medicina no Brasil ante os desafios da formação para a Atenção Primária à Saúde. Saúde debate. 2018; 42 (esp1):189-207.

9. Adler MS, Gallian DMC. Formação médica e serviço único de saúde: propostas e práticas descritas na literatura especializada. Rev. Bras. Educ. Med [internet]. 2014 [acesso em 2018 nov 6]; 38(3):388396. Disponível em: http://dx.doi.org/10.1590/ S0100-55022014000300014.
${ }^{*}$ Orcid (Open Researcher and Contributor ID). 
10. Ceccim RB, Feuerwerker LMC. O quadrilátero da formação para a área da saúde: ensino, gestão, atenção e controle social. Physis. 2004; 14(1):41-65.

11. Vendruscolo C, Ferraz F, Prado ML, et al. Teachingservice integration and its interface in the context of reorienting health education. Interface (Botucatu) [internet]. 2016 [acesso em 2018 nov 6]; 20(59):1015-1025. Disponível: http://dx.doi. org/10.1590/1807-57622015.0768.

12. Vendruscolo C, Prado ML, Kleba ME. Teachingservice integration within the National Professional Health Education Reorientation Program. Ciênc. Saúde Colet. [internet]. 2016 [acesso em 2018 nov 6]; 21(9):2949-2960. Disponível: http://dx.doi. org/10.1590/1413-81232015219.12742015.

13. Cunha PF, Magajewski F. Gestão participativa e valorização dos trabalhadores: avanços no âmbito do SUS. Saúde Soc. [internet]. 2012 [acesso em 2018 nov 6]; 21(supl1):71-79. Disponível em: http://dx.doi. org/10.1590/S0104-12902012000500006.

14. Costa MV, Patrício KP, Câmara AMCS, et al. Pró-Saúde e PET-Saúde como espaços de educação interprofissional. Interface (Botucatu) [internet]. 2015 [acesso em 2018 nov 6]; 19(supl1):709-720. Disponível em: http://dx.doi.org/10.1590/1807-57622014.0994.

15. Lima PAB, Rozendo CA. Desafios e possibilidades no exercício da preceptoria do Pró-PET-Saúde. Interface [internet]. 2015 [acesso em 2018 jul 2]; 19(supl1):779-791. Disponível em: https://doi. org/10.1590/1807-57622014.0542.

16. Pizzinato A, Gustavo AS, Santos BRL, et al. A integração ensino-serviço como estratégia na formação profissional para o SUS. Rev. Bras. Educ. Med. 2012; 36(1)(supl 2):170-177.

17. Jesus JCM, Ribeiro MVB. Uma avaliação do processo de formação pedagógica de preceptores do internato médico. Rev Bras Educ Med. 2012; 36(2):153-161.

18. Faria L, Quaresma MA, Patiño RA, et al. Teachingservice-community integration in practice scenarios of interdisciplinary Health Education: an experience of the Work Education for Health Program (PET-Health) in Southern Bahia. Interface (Botucatu) [internet]. 2018 [acesso em 2018 nov 6]; 22(67):1257-1266. Disponível em: http://dx.doi. org/10.1590/1807-57622017.0226.

19. Ferreira VS, Barreto RLM, Oliveira EK, et al. PETSaúde: uma experiência prática de integração ensino-serviço-comunidade. Rev Bras Educ Med [internet] 2012 [acesso em 2018 nov 6]; 36(1)(supl2):14751. Disponível em: https://dx.doi.org/10.1590/ S0100-55022012000300021.

20. Mira QLM, Barreto RMA, Vasconcelos MIO. Impacto do pet-saúde na formação profissional: uma revisão integrativa. Rev. Baiana Saúde Pública [internet]. 2016 [acesso em 2018 jul 1]; 40(2):514-531. Disponível em: https://doi.org/10.22278/2318-2660.2016.v40. n2.al682.

21. Conselho Nacional de Saúde. Resolução n ${ }^{\circ} 569$, de 8 de dezembro de 2017. Aprova o Parecer Técnico ${ }^{\circ}$ 300/2017. Diário Oficial da União. 26 Fev 2018.

22. Alves CRL, Belisário SA, Lemos JMC, et al. Mudanças curriculares: principais dificuldades na Implementação do PROMED. Rev. Bras. Educ. Med. [internet]. 2013 [acesso em 2018 nov 6];37(2):157-166. Disponível: http:// www.scielo.br/pdf/rbem/v37n2/02.pdf.

23. Bowe CM, Lahey L, Kegan R, et al. Questioning the 'big assumptions'. Part II: recognizing organizational contradictions that impede institutional change. Med. Educ. 2003; 37(8):723-733.

24. Costa NMSC. Docência no ensino médico: por que é tão difícil mudar? Rev. Bras. Educ. Med. 2007; 31(1):21-30.

25. Peduzzi M. Multiprofessional healthcare team: concept and typology. Rev. Saúde Pública [internet]. 2001 [acesso em 2018 nov 6]; 35(1):103109. Disponível em: http://dx.doi.org/10.1590/ S0034-89102001000100016.

26. Oliveira HM, Moretti-Pires RO, Parente RCP. As 
relações de poder em equipe multiprofissional de Saúde da Família segundo um modelo teórico arendtiano. Interface (Botucatu). 2011; 15(37):539-550.

27. World Health Organization. Framework for action on interprofessional education and collaborative practice. Geneva; Switzerland: World Health Organization; 2010.

28. Batista SHSS, Jansen B, Assis EQ, et al. Formação em
Saúde: reflexões a partir dos Programas Pró-Saúde e PET-Saúde. Interface (Botucatu) [internet]. 2015 [acesso em 2018 nov 6]; 19(supl1):743-752. Disponível: http://dx.doi.org/10.1590/1807-57622014.0996.

Recebido em 06/11/2018

Aprovado em 02/02/2019

Conflito de interesses: não houve

Suporte financeiro: a pesquisa da qual derivou este manuscrito

foi financiada via Carta Acordo SCON2017-00547, estabelecida

entre a Organização Pan-Americana da Saúde (Opas) e o Centro

de Estudos e Pesquisa em Saúde Coletiva (Cepesc) 